\title{
ABC of Neo-Newtonian dynamics of static magnetic field
}

This paper comprises upon an illustrated and comprehensive summary of a long experimental research. Not only the research presents a totally new method to study magnetic force but also output of the research is unique. A new branch of science seems to emerge. Full description of the research has been written in 7 draft papers. All the 7 draft papers and a document comprising upon previous discussion are being uploaded, as supplementary files, on the project of this preprint.

\section{Introduction}

I have analyzed the magnetic force between an electromagnet and a permanent magnet in accordance with Newton's first law of motion. The analysis concluded that magnetic force of permanent magnet must work or this force must be balanced (canceled) by an opposing force.

To check the results of the analysis, I have conducted thousands of experiments on the magnetic force. Reasonable amount of opposing force appeared during the experiments. Appearance of the opposing force has validated the results of the analysis.

Moreover, I have successfully reduced the production of the opposing force. Resultant force exceeded to a great extent ultimately. I have made 4 working proofs of the concept. The working proofs of the concept convert the recovered resultant force into additional output power. According to Newton's first law of motion, the emerging of the additional output power is not only absolutely natural but also is mandatory.

\section{Summary of the analysis and results of the experiments}

- Newton's first law of motion is undeniable and applies all kinds of forces. It must also apply on magnetic force. When an electromagnet interacts with a permanent magnet, force of permanent magnet is also present in the system. In accordance with the first law motion, we must calculate magnetic forces of both of the electromagnet and permanent magnet to deduct "potential amount of resultant force". If the same quantity of the potential resultant force does not appear as output power, we must investigate about the lost part of the potential resultant force. If a fraction of potential amount of resultant force disappears, there must be sufficient reason of the disappearance. Once the reason is explored, it must be investigated whether the elimination of the reason is possible or not.

- I have experimentally investigated into the matter for a long time. Substantial amount of extra efficiency emerged during experiments on repulsion and attraction forces between an electromagnet and a permanent magnet. Repeatedly repeatable experiments verified emerging of this extra efficiency undeniably. Whenever both current (of electromagnet) and flux (of permanent magnet) were enhanced, efficiency jumped higher. Output must increase because of enhanced current and flux but from where does the extra efficiency come? $\mathrm{I}^{\wedge} 2 \mathrm{R}$ loss increases as a result of increment in current so efficiency must drop but it increases instead! No rule of 
electromagnetism justifies this extra efficiency. Only the first law of motion justifies the extra efficiency sufficiently.

- While analyzing the results of the experiments, I found that a fraction of the iron core of electromagnet always remains unsaturated. During repulsion, permanent magnet repels like pole of electromagnet and attracts the unsaturated part of the iron core simultaneously. This phenomenon may be named as "attraction during repulsion" or "remnant attraction".

- This two-fold action of permanent magnet balance (cancels) a huge part of repulsion force. In other words, permanent magnet causes matching and opposing forces simultaneously that cancel each other. Since, a complete cycle of repulsion and attraction is needed for work, the severe damage of repulsion force results in heavy loss of output power of a mechanical interaction between an electromagnet and a permanent magnet.

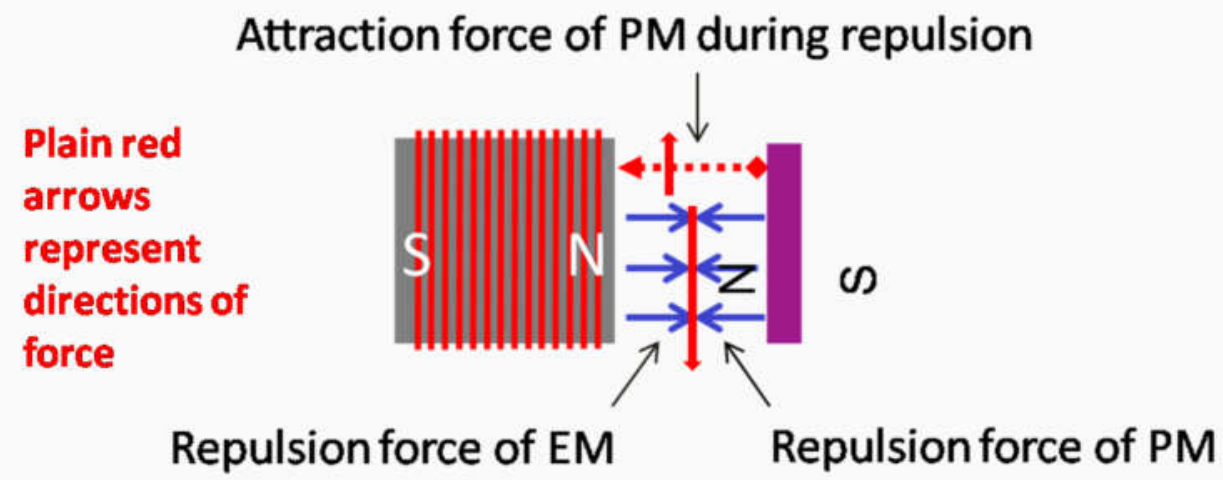

- Basic reason of the damage is the presence of unsaturated part of iron core. It is obvious that at higher degree of magnetization of the iron core, this unsaturated part reduces, and at lower degree of magnetization this part expands. So basic remedy to the damage of the output power is increment in degree of magnetization of the iron core. For enhancement of the degree of magnetization, current, turns of coil or both of them can be used.

- More permeability cannot be used to enhance the magnetic field. Adding of more permeability will enlarge the unsaturated part that will produce bigger opposite force. It will end in heavy loss of output power.

However, it is not always possible to saturate the iron core. To overcome this hindrance, I have developed at least 4 most efficient alternative techniques. These techniques reduce the negative factor "attraction during repulsion" greatly. Resultant force becomes substantially stronger because of reduction of the negative factor. Output power of a mechanical interaction between an electromagnet and a permanent magnet becomes substantially stronger in the end.

\section{Working proof of concept 1: High Repulsion Permanent Magnet}

- During the research a new kind of permanent magnet emerged. I have named this kind of permanent magnet as High Repulsion/ Permeable permanent magnet. Making process of this magnet is simple but function of this magnet is very important.

- A piece of non -magnetized material was added to the interacting surface of a permanent magnet, then the magnet was interacted with the same electromagnet that was interacted with an ordinary permanent magnet. Addition of permeability to a permanent magnet provided marvelous results. 
- Permeability added permanent magnet provides significantly more repulsion force than ordinary permanent magnet using the same input current, at lower current levels; while attraction forces of both kinds of permanent magnets remain the same.

\section{Repulsion between a permeable permanent magnet and an electromagnet}

- When a permeable permanent magnet repels an electromagnet, some of its flux repels the flux of the electromagnet.

- Some of its flux emits from sides of iron piece to avoid confrontation to the like flux of the electromagnet.

- Only a smaller quantity of flux remains available to penetrate into the electromagnet.

- Weak penetration of PM flux into iron of the electromagnet produces weak "attraction during repulsion".

- Weak attraction balances smaller part of repulsion force.

- So, repulsion force of permeable permanent magnet is always stronger than that of an ordinary permanent magnet.

- Figure is given bellow.

Inner set of B lines is making loop with its other pole, avoiding confrontation with opposite like pole.

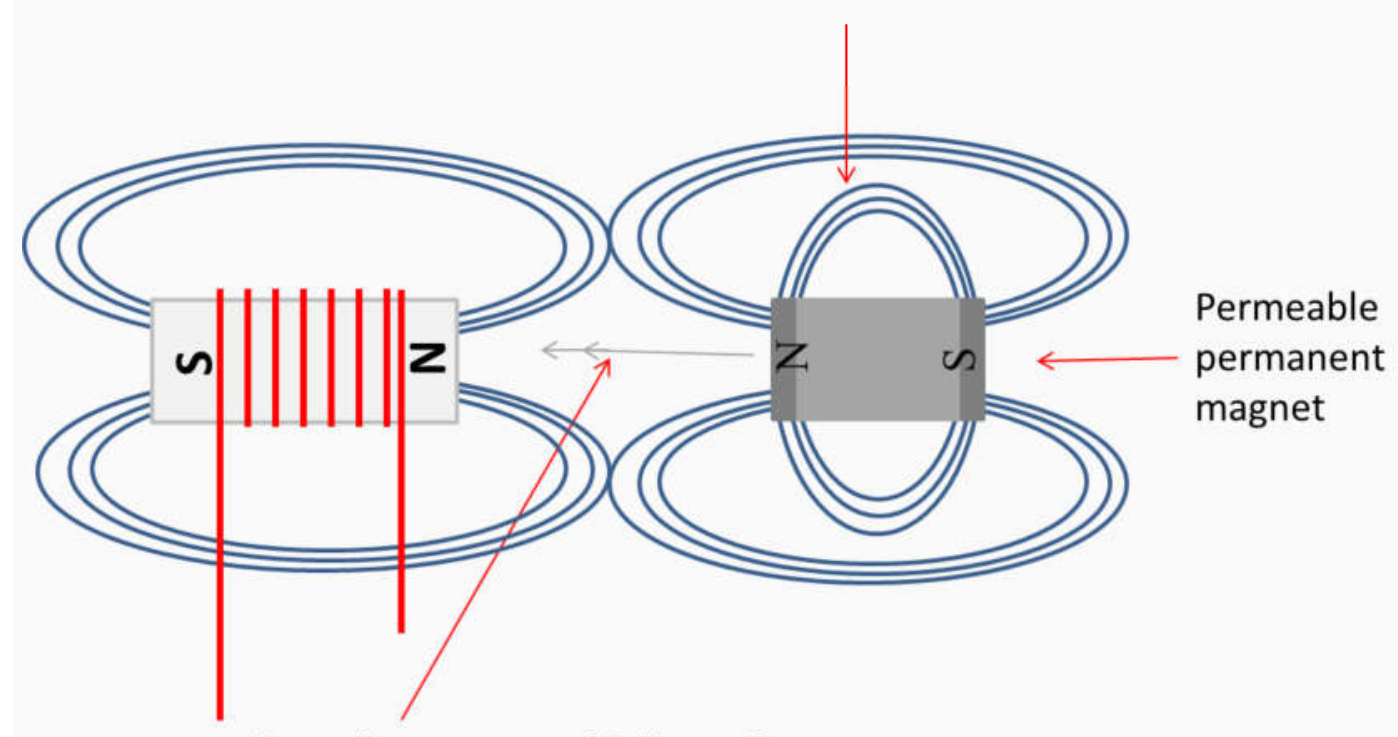

A smaller amount of B lines of outer set is available to penetrate into the iron of electromagnet during repulsion. 
Attraction between a permeable permanent magnet and an electromagnet magnet

- Attraction force of a permeable permanent magnet remains same as that of an ordinary permanent magnet.

- Inner set of flux lines ceases to exist due to presence of unlike pole on opposite side.

- All B lines follow attraction. So, full attraction force of a permeable permanent magnet is got.

- Figure is given bellow.

Inner set of B lines has been

vanished and all $B$ lines of

permeable permanent magnet

are participating in attraction

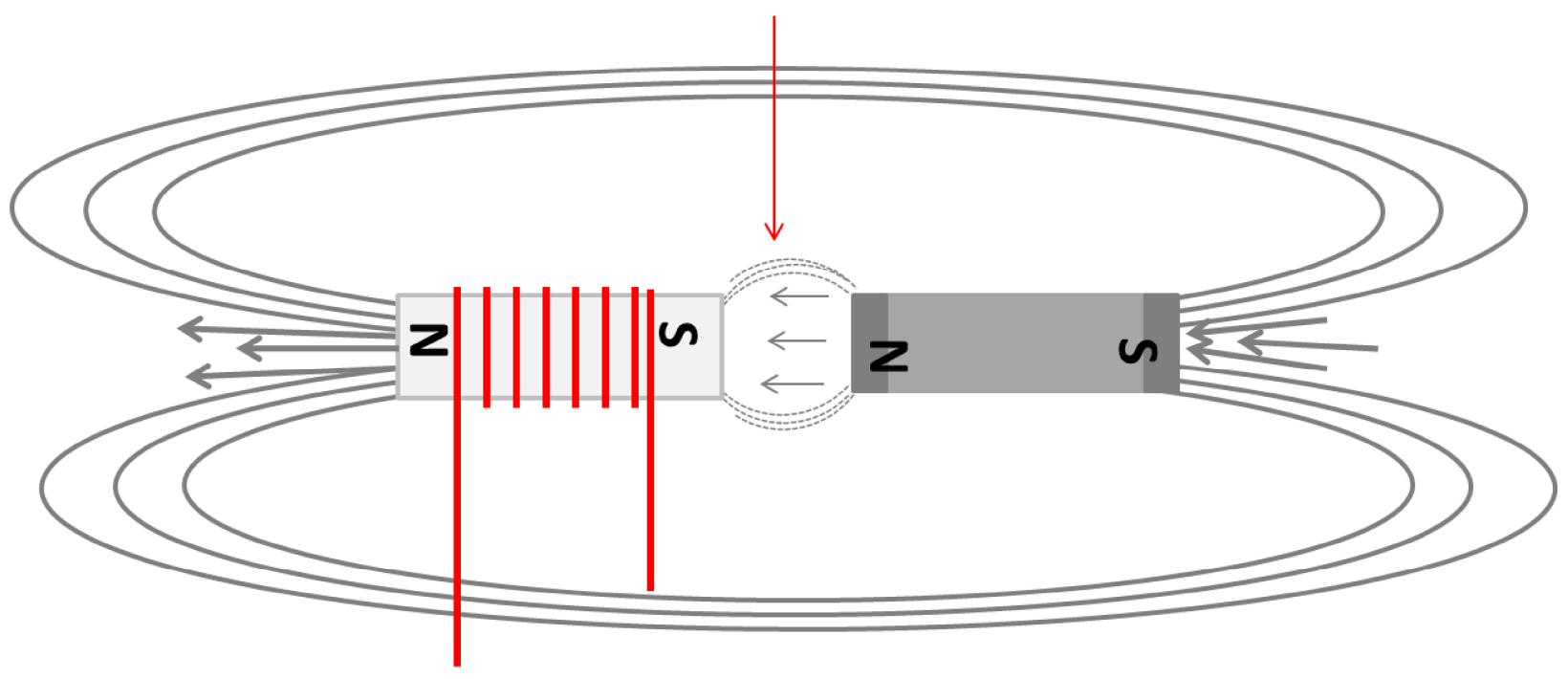

\section{Working proof of concept 2: 12-PM poles and 12- armature-teeth DC motor}

- When both current and PM flux are enhanced in this motor, efficiency increases accordingly, continuously and substantially. Recovery of ever increasing extra efficiency makes this motor most important. Ever increasing efficiency means that an unknown part of the efficiency still remains hidden. Improved models would recover the rest efficiency.

- This is a new phenomenon contrary to present PM motor technology. Present PM motor technology is merely a set of techniques adopted to fulfill practical purpose. In the way of recovery of maximum efficiency, the laws of motion reject this set of techniques. No set of techniques can overcome the laws of motion. 
- Where this extraordinary efficiency is coming from? The results of basic experiments justified by the laws of motion validate this extra efficiency undeniably.

Diagram:

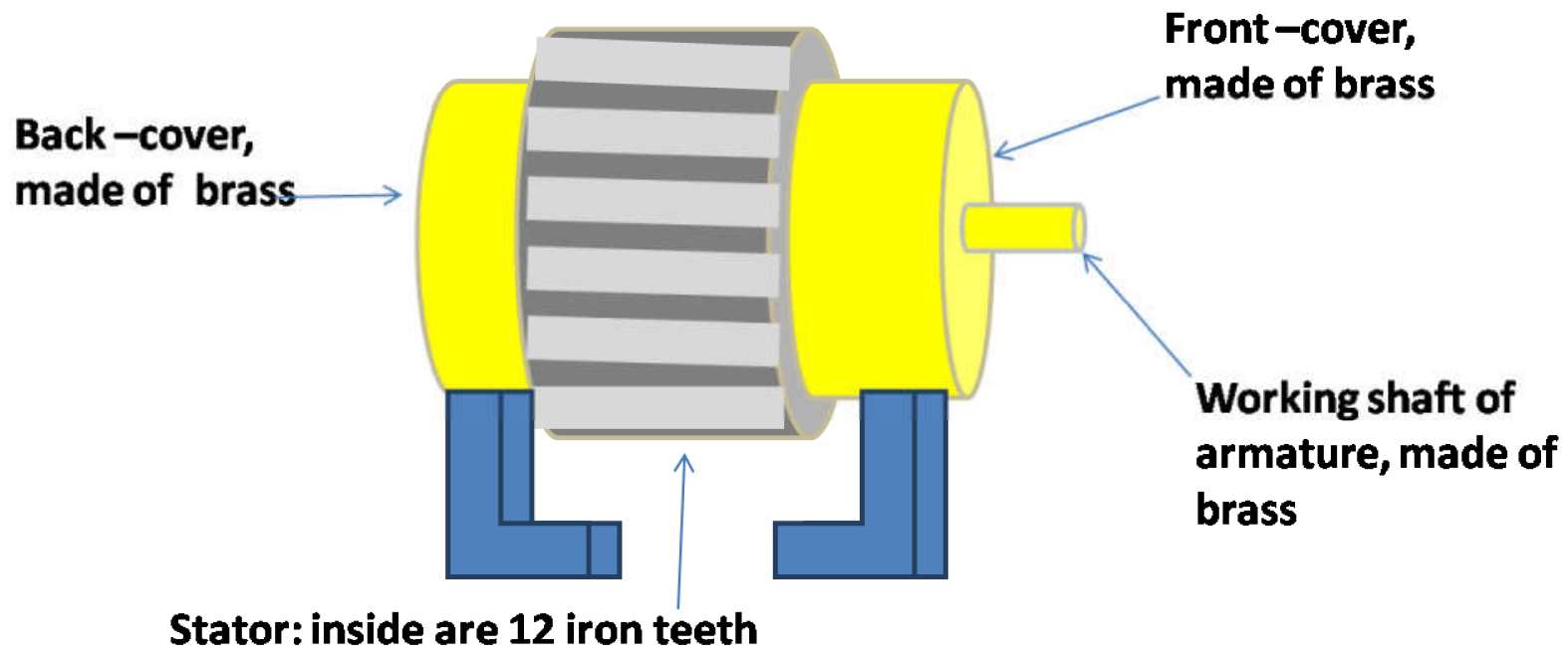

Outside are 12 saturated permanent magnets

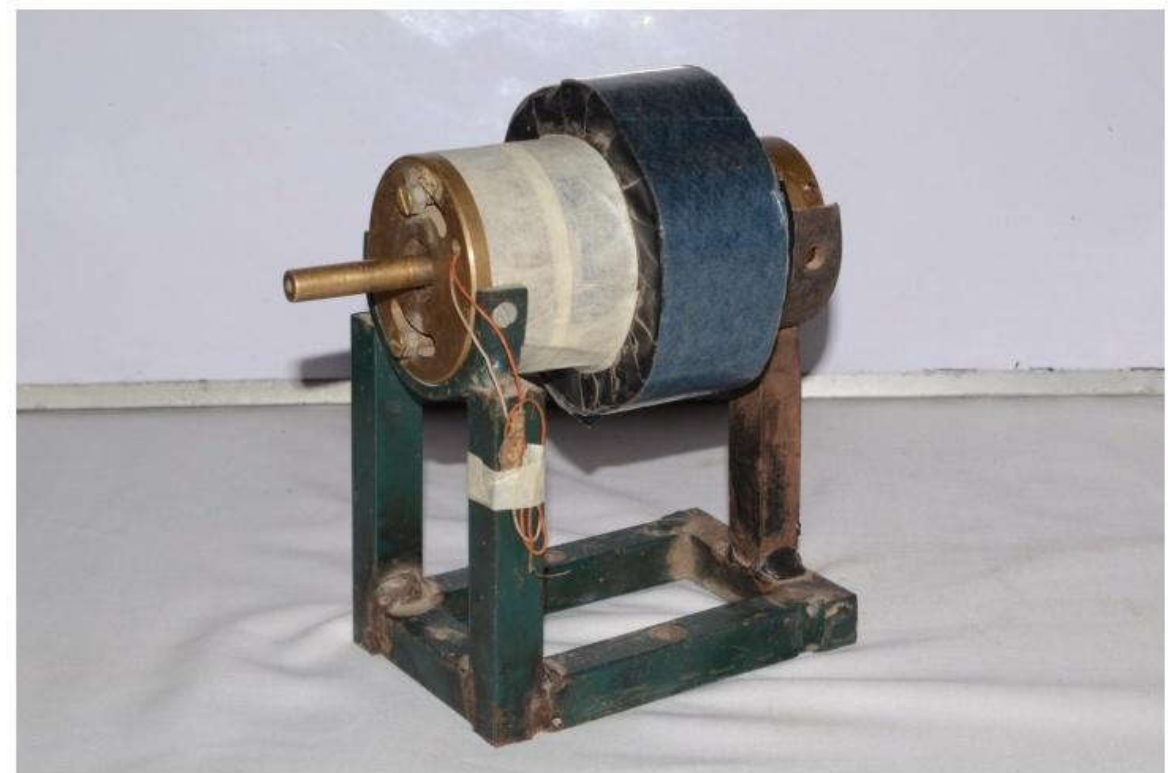

Picture of the motor 


\section{Working proof of concept 3: Integrate magnetic circuit}

Video: https://www.youtube.com/watch?v=y-vUfBMfqoI

This is a rotary actuator.

- An electromagnet moves over two permanent magnet field poles.

- An additional permanent magnet is installed on the moving electromagnet.

- When polarity of the electromagnet is changed, the additional permanent magnet rotates.

- This rotating permanent magnet is removable.

\section{Integrated magnetic circuit}

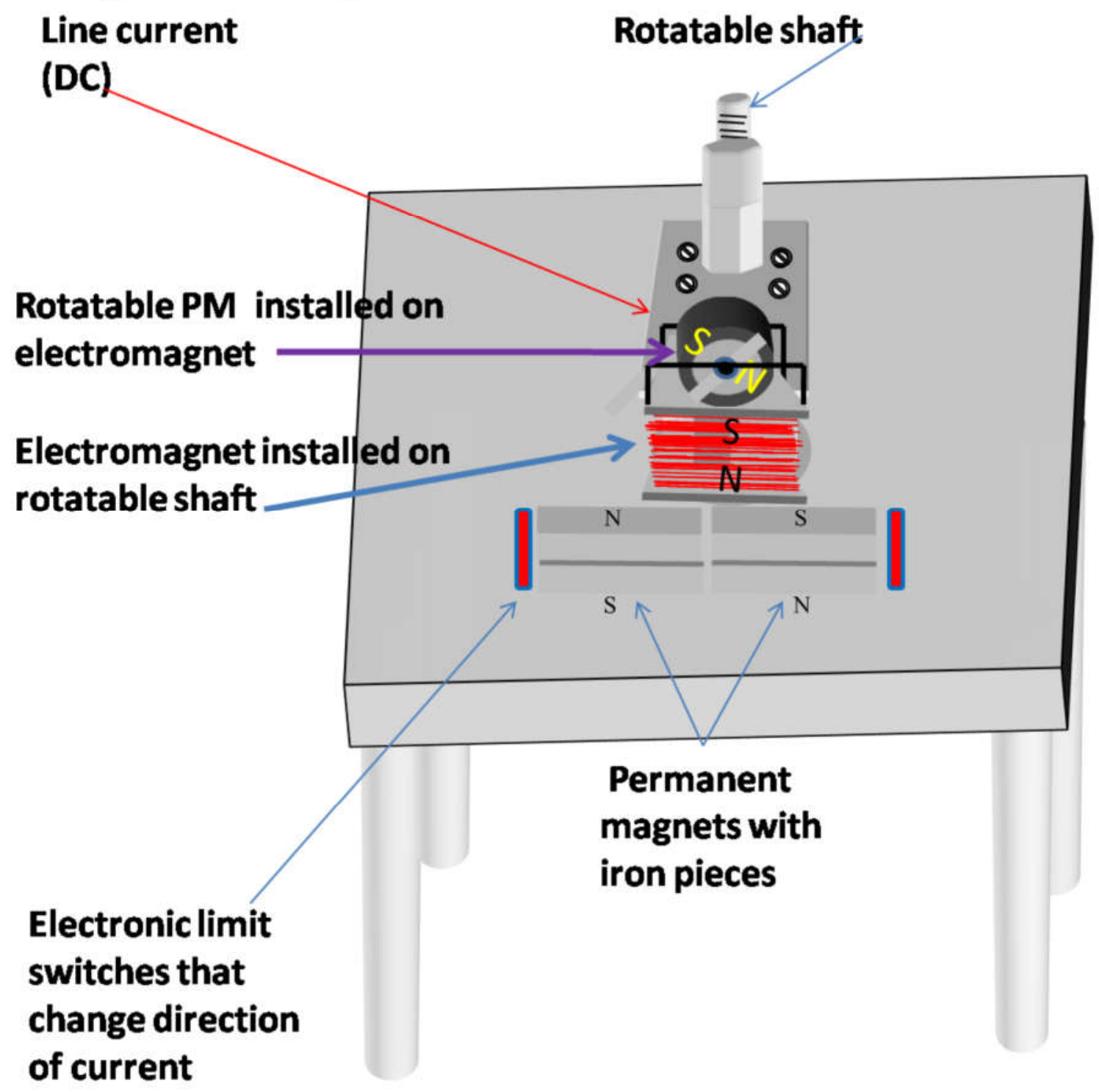


Movement of electromagnet from left to right

Rotating magnet is strengthening North pole of electromagnet.

Direction of movement of electromagnet

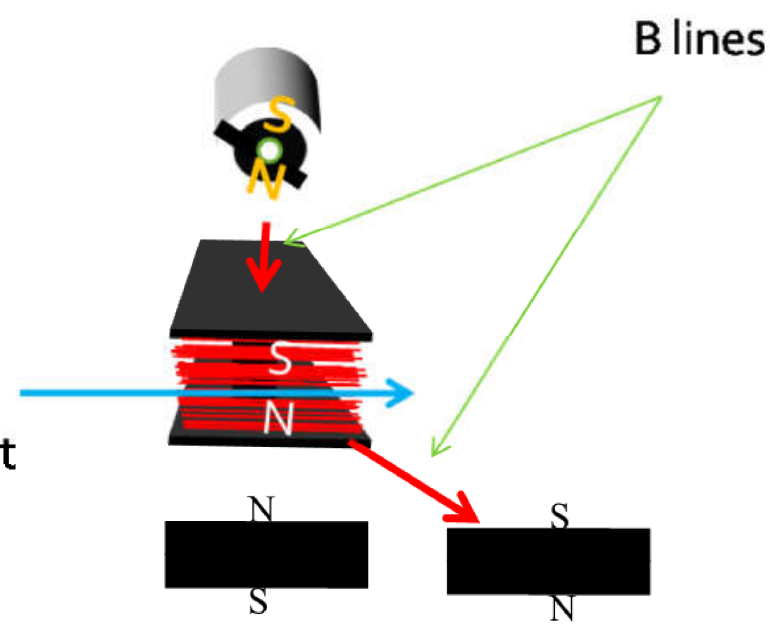

Movement of electromagnet from right to left (when direction of current has been changed).

Rotating magnet is strengthening south pole of electromagnet.

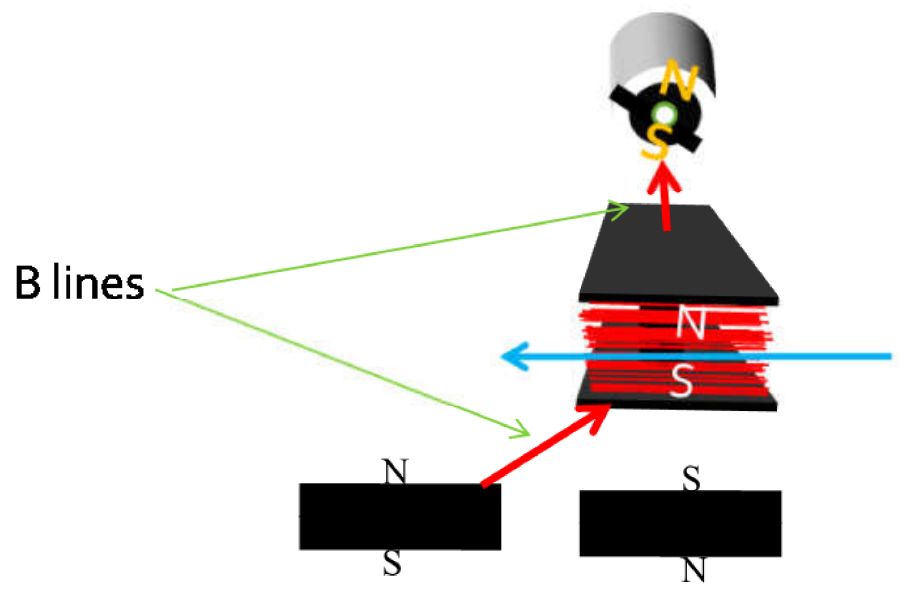

Direction of movement of electromagnet 


\section{Function of the actuator without the rotating permanent magnet}

when the rotating permanent magnet is not installed on the electromagnet, a particular amount of efficiency is acquired using a particular amount of input electricity. Figure:

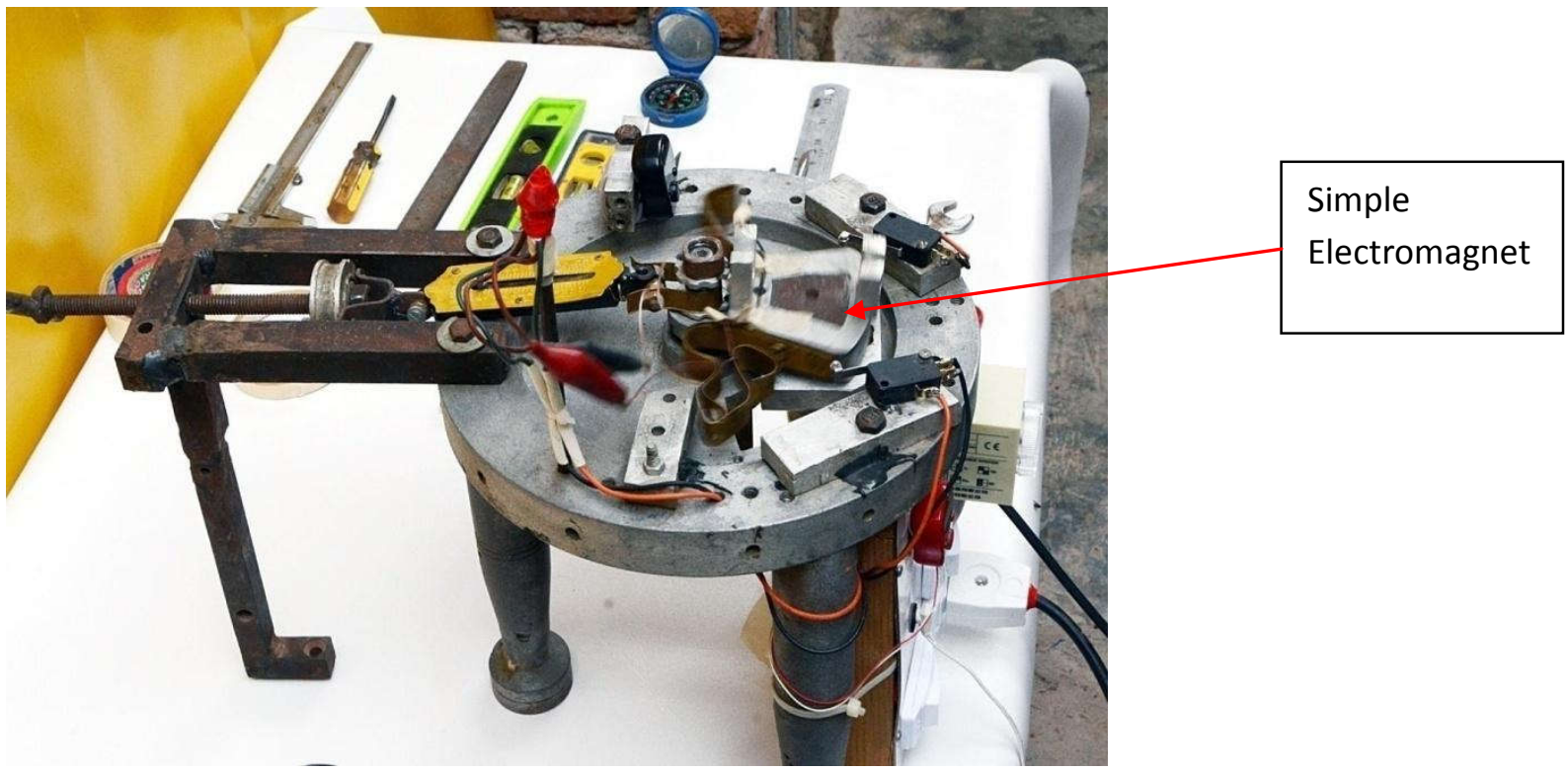

Unique function of rotating permanent magnet:

When the rotating permanent magnet is installed on the electromagnet, efficiency of the actuator jumps $60 \%$ higher using the same amount of input electricity. Figure:
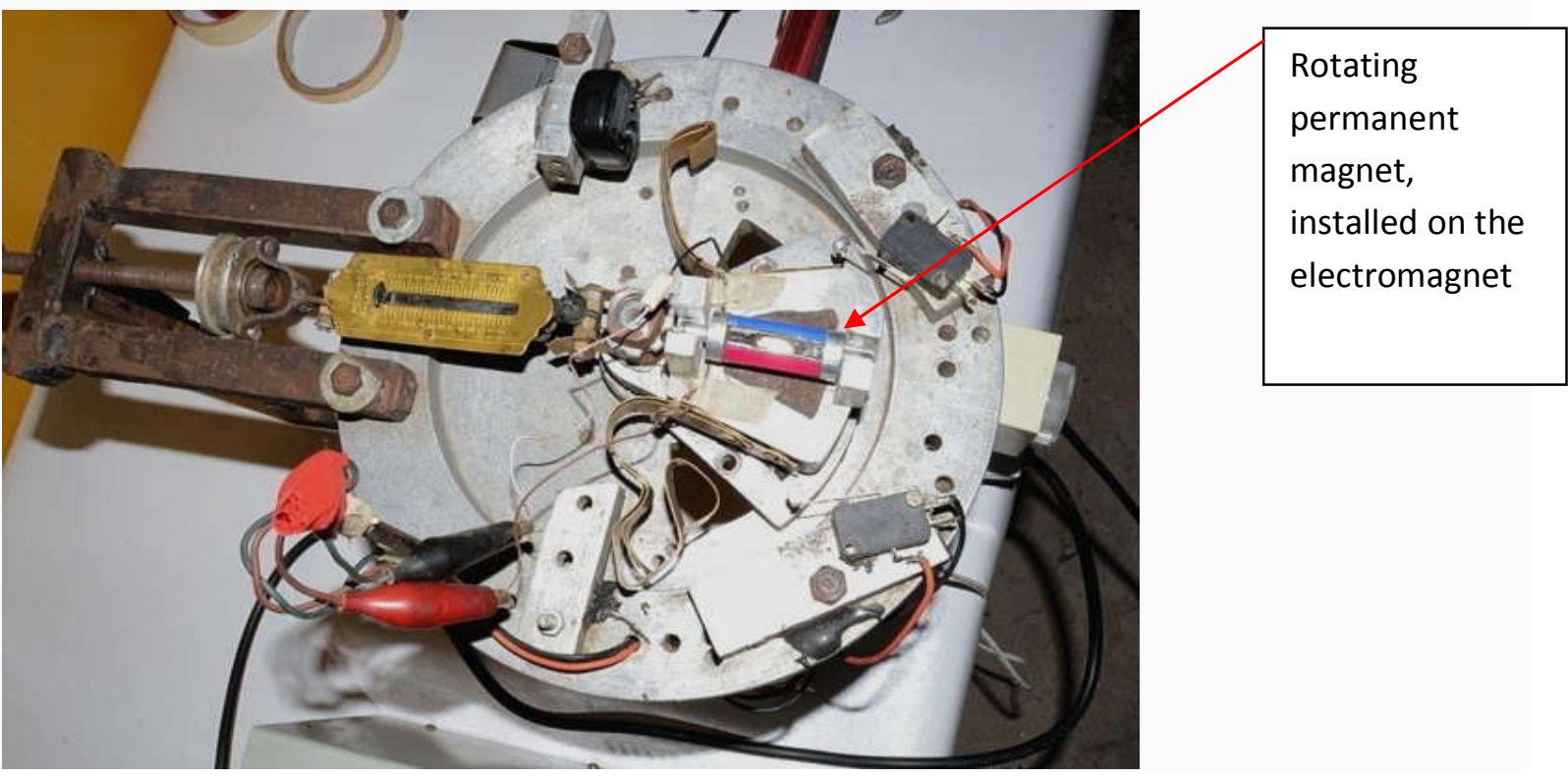

Difference of the efficiencies has been measured comparatively. Rotating magnet provides $60 \%$ more efficiency. 


\section{Working proof of concept 4: Like-pole PM rotary actuator}

\section{Video: $\underline{\text { https://drive.google.com/open?id=1ys1BZL9N7Fb zbEOXJzksRR-00CY7Gvv }}$}

A rotary actuator has been made using only like poles of permanent magnet. Like poles of permanent magnet have not been used in this actuator baselessly. Instead, this usage has concrete basis.

In PM DC motors, because of present designs, permanent magnet causes matching and opposing force simultaneously. This double action balances all force of permanent magnet.

To lessen the balance of force, permanent magnets have been used in this actuator independently. In this actuator, each of two sets of permanent magnets either attracts or repels a pole of electromagnet. So, no double action is produced by permanent magnet. Resultantly, no opposing force is produced.

However, some minor factors still balance a part of magnetic force of permanent magnet, but this minor balance is far lesser than full balance of PM force occurring in present PM DC motors.

\section{This device comprises upon two main components: stator and rotor}

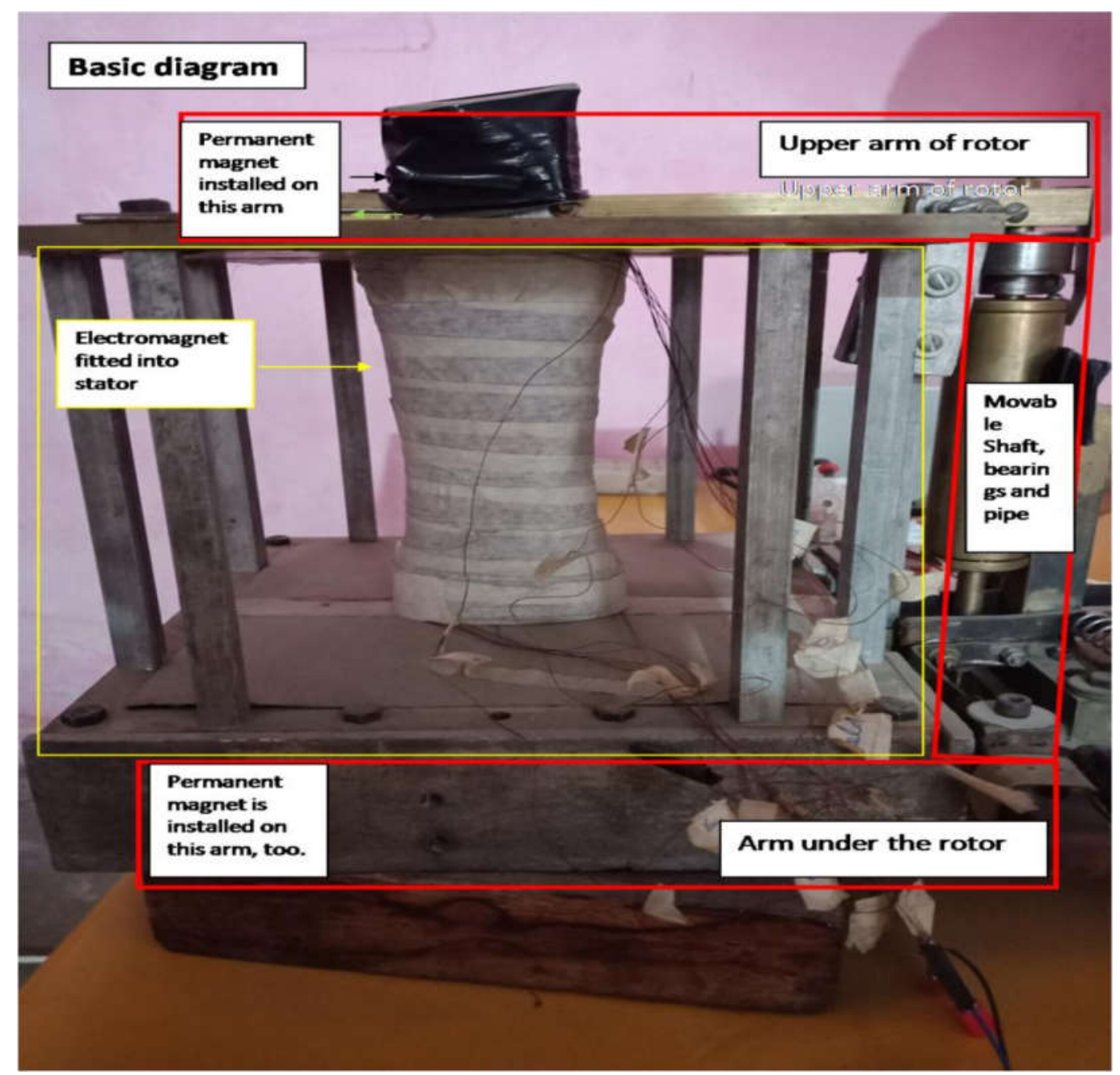


full sketch of rotor with permanent magnets interacting with electromagnet that is situated into the stator

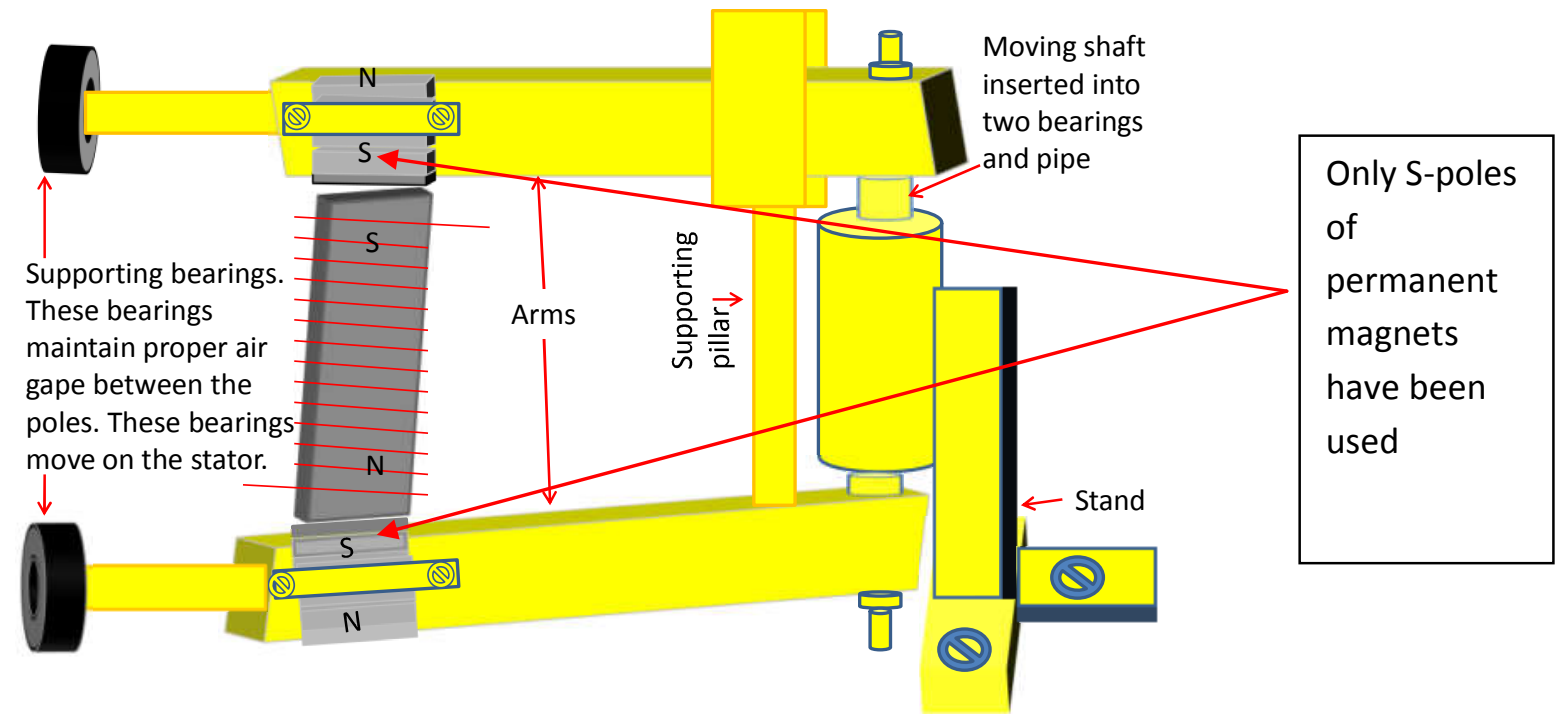

\section{Possible role of force of permanent magnet in building output power}

One of the both set of the permanent magnets repels the other set of permanent magnets and attracts the electromagnet simultaneously. This simultaneous repulsion and attraction is potentially basic source of output power of this machine. Figures:

- Red arrows represent force of permanent magnet

- Black arrows represent force of electromagnet

\section{Role of force of permanent magnet during movement of rotor from right to left}

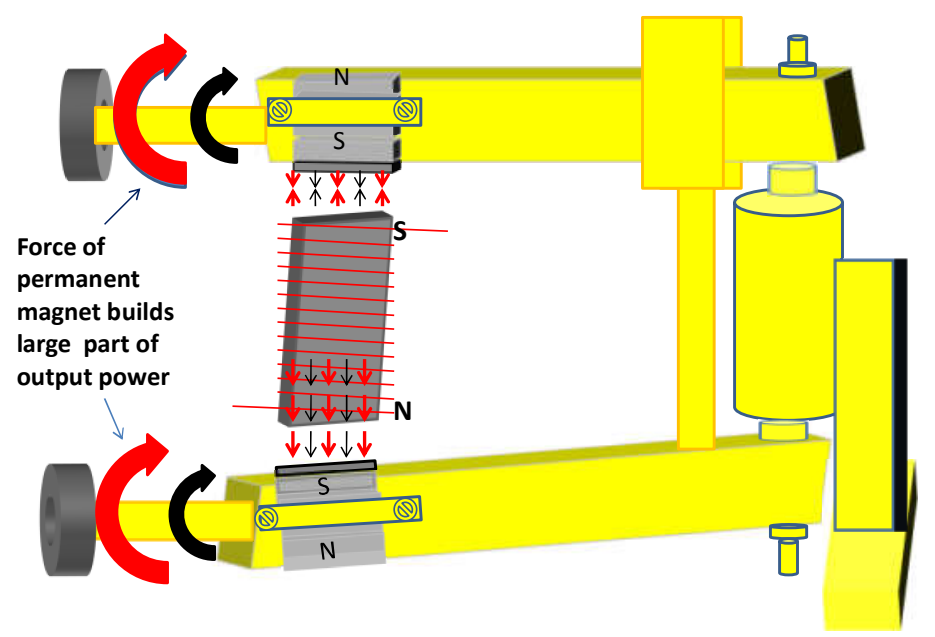




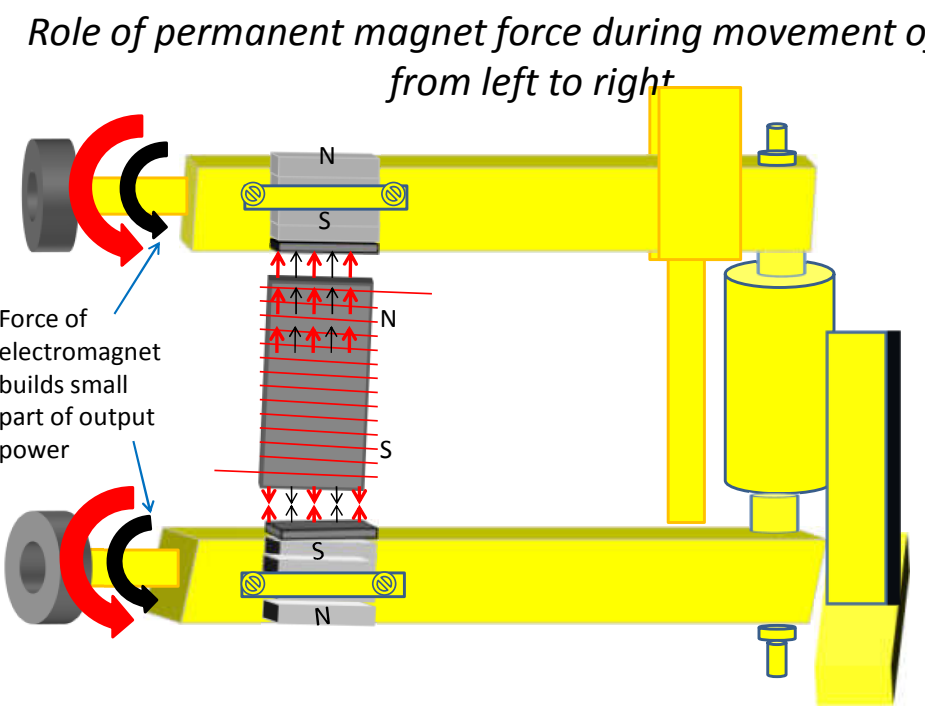

According to early measurements, this machine seems to extract a substantial amount of pm energy. However, regular measurements are underway.

\section{Balance (cancelation) of magnetic force in PM DC motors}

(Balance of force means when two forces act in opposite direction and neutralizes each other's affect. In simple words, balance of force means wastage of force)

This simulation characterizes present understanding of lines of force in PM DC motors.

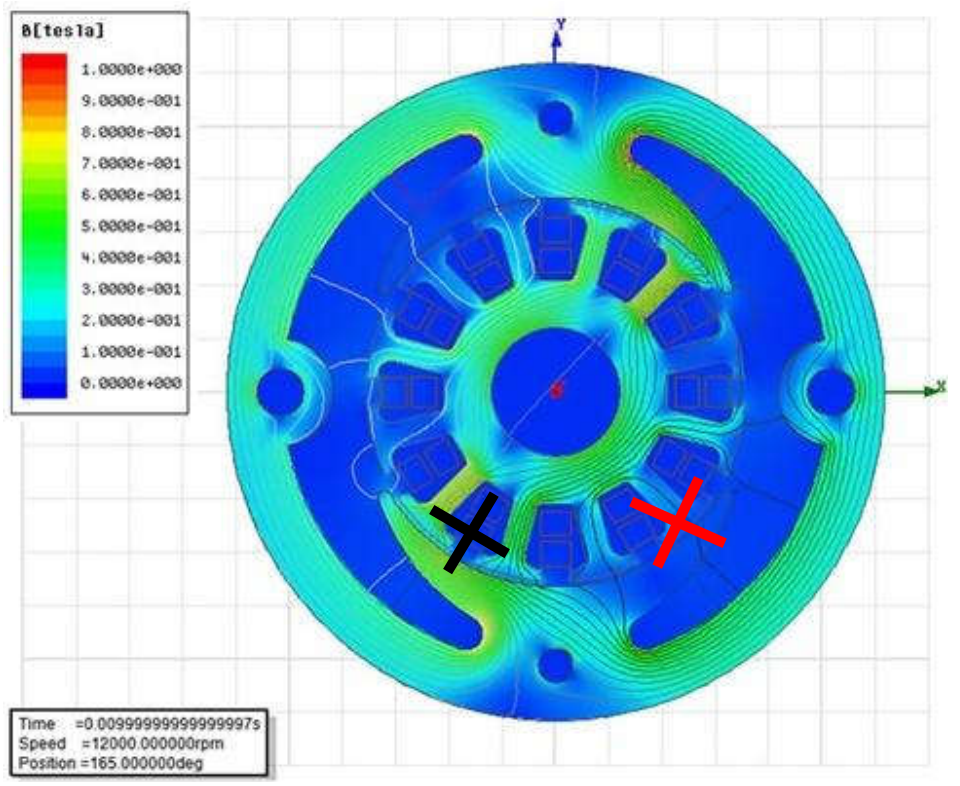




\section{How is it possible?}

Why is half of the field pole (in red crossed area) almost free of lines of force? It is impossible. This portion must contain substantial amount of lines of force.

Lines of force emanating from red crossed area of the field pole can never reach black crossed area because of circular shape of field pole and intervening un-energized armature teeth.

These Lines of force must penetrate into intervening un-energized armature teeth. This penetration must cause heavy attraction against direction of rotation; hence this attraction is heavy opposing force.

This heavy opposing force must balance equivalent part of matching force. In simple words, enormous amount of magnetic force must be wasted because of production of the opposing force.

The understanding represented by the simulation is totally flawed. This flawed understanding is constantly leading to wrong designs of PM DC motors.

\section{Violation of a basic rule of electromagnetism in the computer simulation}

Scientifically, above mentioned computer simulation violates a basic role of electromagnetism, so presents a totally incorrect pattern of field lines.

The violated role:

"Not only B lines emanating from like poles of two permanent magnets repel each other but also B lines emanating from the same pole of a permanent magnet repel each other".

Since B lines emanating from the same pole repel each other they can never join each other to reach the active corner of the pole (red crossed area of the simulation).

Figure

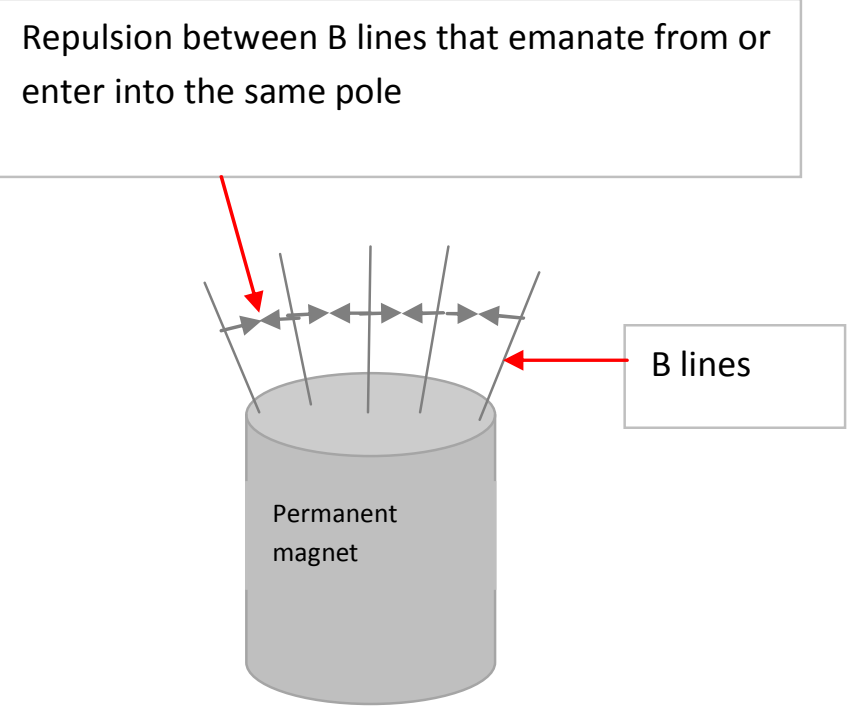


All or most of PM flux cannot concentrate on active corner of the permanent magnet (black circled area) because the role prohibits it.

In other words, if all the field lines have to reach the nearest unlike pole ( armature pole) through black circled area of the permanent magnet, the entire field lines have to lost their basic property of mutual repulsion. This is impossible.

Moreover, it is clearly experimental that when unlike pole of a powerful permanent magnet is attached to one corner of a field-pole; the other corner still attracts a piece of iron forcefully.

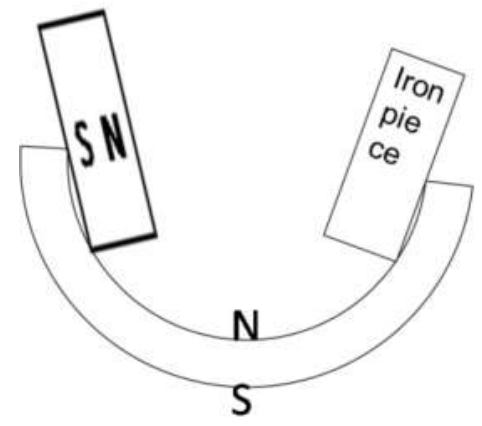

If most of B lines of a field-pole concentrate on active corner, how other corner can attract iron forcefully?

Real pattern of lines of force and directions of forces

\section{Example: BALANCE OF FORCE IN A TWO- POLE PM DC MOTOR}

Figure: 1

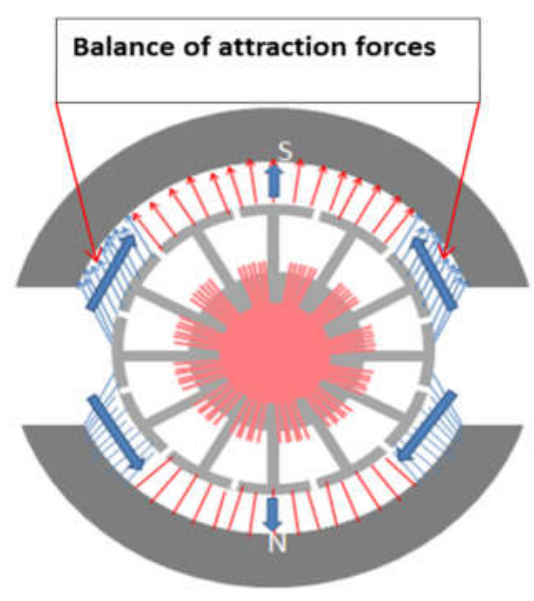

When the motor is at rest (no current is applied):

Substantial force is already present in this motor. Magnetic field of permanent magnet created this force.

Only attraction force is produced in this situation.

However, all this attraction force is not produced in the same direction.

B lines make several different groups. These groups produce opposing attraction forces.

Opposing attraction forces balance each other equally. Motor stays at rest. 


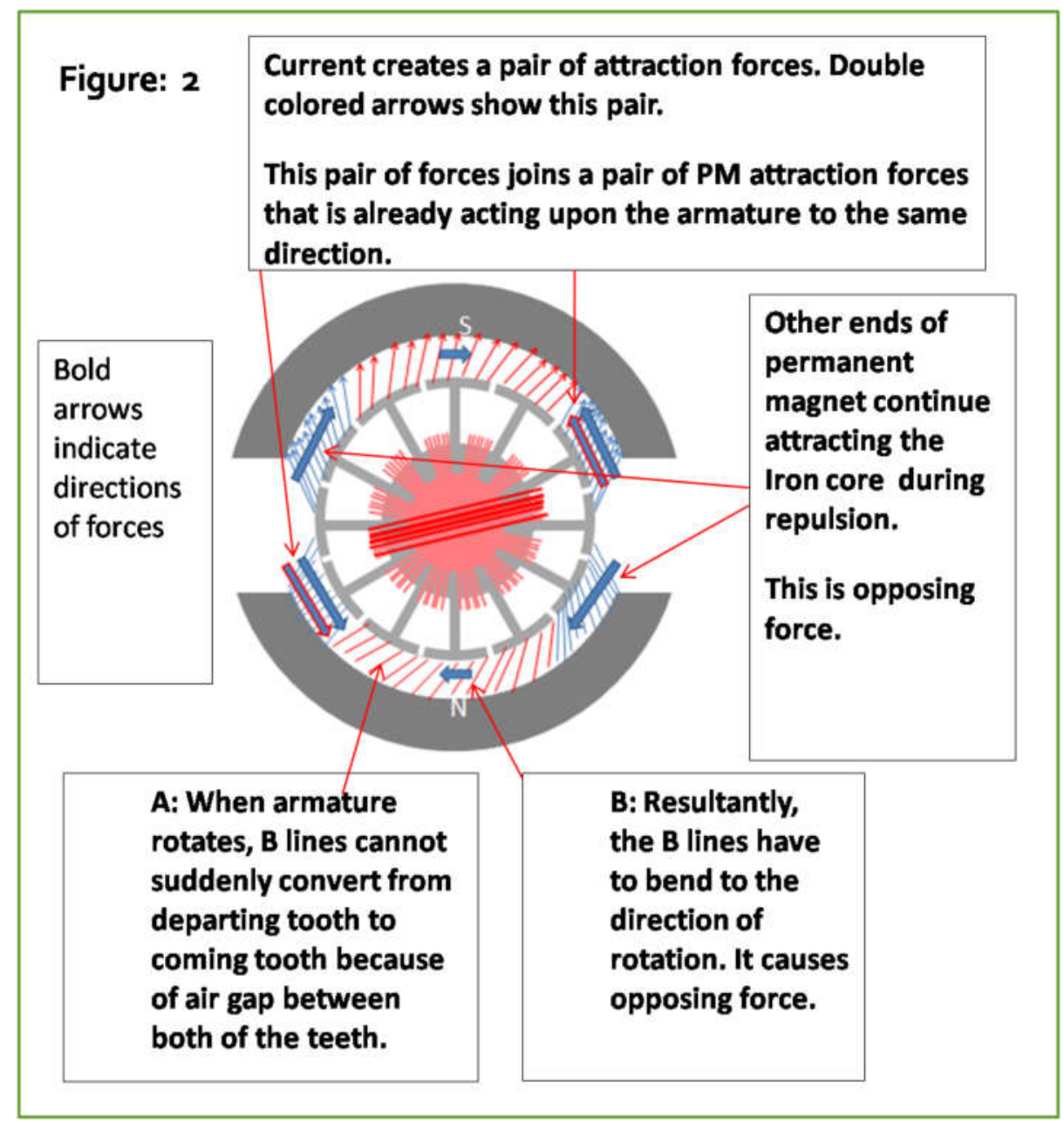

\section{Detail}

- In present designs of PM DC motors, permanent magnet field poles produce heavy matching and heavy opposing forces simultaneously. Both of the matching and opposing forces balance each other. Heavy force is wasted in PM DC motor

- First half of the field pole: matching force of attraction created by current is doubled because matching force of attraction produced by permanent magnet joins it.

- Opposing force created by other half of the field pole balances half of the doubled matching force.

- Only matching force created by current remains available to produce output. 


\section{Result}

Present interpretation of static magnetic field in physics is insufficient and misleading.

The term 'static magnetic field', generally, stands for a magnetic field that is stationary and motionless. The term also results in, "magnetic flux lines ' $B$ ' (of a static magnetic field) have nothing to do with 'work".

In actual fact, as soon as a magnet or ferromagnetic material is inserted into the so-called static magnetic field, the field breaks into many parts. In other words, ' $B$ ' lines divide themselves into many groups. In this situation, every one of the groups has its own intensity and direction. If not controlled, these groups cause opposite forces; if controlled, these groups enhance resultant force to a great extent. 